\title{
Comparison Between Magnetic Resonance Imaging and Computed Tomography of the Lung in Patients With Cystic Fibrosis With Regard to Clinical, Laboratory, and Pulmonary Functional Parameters
}

\author{
Diane M. Renz, MD, * Oriane Scholz, MD, * Joachim Böttcher, MD, † Martin H. Maurer, MD, * \\ Timm Denecke, MD, * Carsten Schwarz, MD, $\neq$ Alexander Pfeil, MD, $\S$ Florian Streitparth, MD, * \\ Alexander Huppertz, MD, * Anne Mehl, MD, $\neq$ Alexander Poellinger, MD, * Doris Staab, MD, $\neq$ \\ Bernd Hamm, MD, * and Hans-Joachim Mentzel, MD\|
}

\begin{abstract}
Objective: To evaluate whether magnetic resonance imaging (MRI) is effective as computed tomography $(\mathrm{CT})$ in determining morphologic and functional pulmonary changes in patients with cystic fibrosis (CF) in association with multiple clinical parameters.

Materials and Methods: Institutional review board approval and patient written informed consent were obtained. In this prospective study, 30 patients with CF (17 men and 13 women; mean (SD) age, 30.2 (9.2) years; range, 19-52 years) were included. Chest $\mathrm{CT}$ was acquired by unenhanced low-dose technique for clinical purposes. Lung MRI (1.5 T) comprised T2- and T1-weighted sequences before and after the application of $0.1-\mathrm{mmol} \cdot \mathrm{kg}^{-1}$ gadobutrol, also considering lung perfusion imaging. All CT and MR images were visually evaluated by using 2 different scoring systems: the modified Helbich and the Eichinger scores. Signal intensity of the peribronchial walls and detected mucus on T2-weighted images as well as signal enhancement of the peribronchial walls on contrast-enhanced T1-weighted sequences were additionally assessed on MRI. For the clinical evaluation, the pulmonary exacerbation rate, laboratory, and pulmonary functional parameters were determined.

Results: The overall modified Helbich CT score had a mean (SD) of 15.3 (4.8) (range, 3-21) and median of 16.0 (interquartile range [IQR], 6.3). The overall modified Helbich MR score showed slightly, not significantly, lower values (Wilcoxon rank sum test and Student $t$ test; $P>0.05$ ): mean (SD) of 14.3 (4.7) (range, 3-20) and median of 15.0 (IQR, 7.3). Without assessment of perfusion, the overall Eichinger score resulted in the following values for CT vs MR examinations: mean (SD), 20.3 (7.2) (range, 4-31); and median, 21.0 (IQR, 9.5) vs mean (SD), 19.5 (7.1) (range, 4-33); and median, 20.0 (IQR, 9.0). All differences between CT and MR examinations were not significant (Wilcoxon rank sum tests and Student $t$ tests; $P>0.05$ ). In general, the correlations of the $\mathrm{CT}$ scores (overall and different imaging parameters) to the clinical parameters were slightly higher compared to the MRI scores. However, if all additional MRI parameters were integrated into the scoring systems, the correlations reached the values of the CT scores. The overall image quality was significantly higher for the CT examinations compared to the MRI sequences.
\end{abstract}

Received for publication February 28, 2015; and accepted for publication, after revision, April 24, 2015

From the *Department of Radiology, Charité University Medicine Berlin, Campus Virchow Clinic, Berlin; † Institute of Diagnostic and Interventional Radiology, SRH Clinic Gera, Gera; †Division of Pulmonology and Immunology, Department of Pediatrics, Charité University Medicine Berlin, Campus Virchow Clinic, Berlin; $\S$ Department of Internal Medicine III, and \|Institute of Diagnostic and Interventional Radiology, Department of Pediatric Radiology, Friedrich-SchillerUniversity, Jena University Hospital, Jena, Germany.

Conflicts of interest and sources of funding: Supported by a research grant of Bayer HealthCare Pharmaceuticals, Berlin, Germany. However, the data and the results of the study were independently obtained, and the investigators had exclusive control of all data.

The authors report no conflicts of interest.

Correspondence to: Diane M. Renz, MD, Department of Radiology, Charité University Medicine Berlin, Campus Virchow Clinic, Augustenburger Platz 1, 13353 Berlin, Germany. E-mail: diane.renz@charite.de.

Copyright (C) 2015 Wolters Kluwer Health, Inc. All rights reserved.

ISSN: $0020-9996 / 15 / 5010-0733$

DOI: $10.1097 /$ RLI.0000000000000178
Conclusions: One major diagnostic benefit of lung MRI in CF is the possible acquisition of several different morphologic and functional imaging features without the use of any radiation exposure. Lung MRI shows reliable associations with CT and clinical parameters, which suggests its implementation in CF for routine diagnosis, which would be particularly important in follow-up imaging over the long term.

Key Words: cystic fibrosis, magnetic resonance imaging, computed tomography, clinical parameters, pulmonary functional test

(Invest Radiol 2015;50: 733-742)

ystic fibrosis (CF) is the most prevalent autosomal recessive disease in the white population. ${ }^{1,2}$ This inherited progressive disease is caused by mutations of the $\mathrm{CF}$ transmembrane conductance regulator gene localized on the long arm of chromosome $7 .{ }^{1}$ Various possible mutations of this gene result in an impaired transepithelial chloride secretion in sweat, salivary, and intestinal glands as well as in the airways. ${ }^{1,2}$ Pulmonary manifestations are responsible for more than $90 \%$ of the morbidity and mortality in patients with $\mathrm{CF}^{2-4}$ Owing to advances in management and treatment of these pulmonary manifestations, the life expectancy of patients is continuously increasing, with a current median survival of approximately 40 years. ${ }^{3-5}$

Accurate diagnosis and monitoring of CF-related lung disease is crucial in providing early and effective treatment and also in improving the overall prognosis. Thin-sliced chest computed tomography (CT) is the current criterion standard to visualize best the characteristic morphologic pulmonary changes due to its high spatial resolution. ${ }^{6-8}$ Thereby, scoring systems offer a quantitative and more reproducible assessment compared to sole visual imaging analysis. ${ }^{8-10}$ As repeated, life-long pulmonary imaging is required, the cumulative radiation exposure can itself become a complication for patients with $\mathrm{CF}$, particularly with regard to their increasing life expectancy, even if low-dose CT protocols are used; in the case of 1 to $2 \mathrm{CT}$ examinations per year, cumulative radiation doses of more than $40 \mathrm{mSv}$ can be achieved. ${ }^{8-11}$

Magnetic resonance imaging (MRI) of the lung has increasingly been proposed as a radiation-free diagnostic alternative in patients with CF. ${ }^{12-14}$ In 1987, Fiel et al ${ }^{15}$ first described the use of lung MRI in young adults with CF. However, the early investigations on lung MRI could not provide comparable findings to CT examinations. ${ }^{15,16}$ With the development and ongoing improvement of MRI sequences and equipment, such as multichannel radiofrequency coils, the temporal and spatial resolution of lung MRI has been continuously optimized. ${ }^{17-20}$ Puderbach et $\mathrm{al}^{12}$ found high agreement of morphologic pulmonary changes between MRI and CT in CF. Although spatial resolution of MRI is still inferior compared to CT, MRI has the advantage of assessing morphologic and functional aspects of lung parenchyma without any ionizing radiation; furthermore, MRI can provide distinct tissue characterization based on signal intensities in T1- and T2-weighted imaging as well as enhancement features after contrast medium application. ${ }^{12,21-24}$ 
The principle aim of this investigation was to determine the diagnostic value of MRI to visualize pulmonary manifestations in patients with CF compared to unenhanced low-dose CT and, moreover, to clinical findings. The main difference between this prospective study and published investigations is the cross-sectional correlation of all imaging results to laboratory and pulmonary functional parameters as well as to possible pulmonary exacerbation. Furthermore, the overall image quality of the CT and MR examinations was assessed. All CT series and MRI sequences were evaluated using 2 different scoring systems to find the best possible evaluation score for CT and MR images.

\section{MATERIALS AND METHODS}

\section{Study Standards and Design}

This prospective investigation was performed in accordance with the Declaration of Helsinki and was approved by the local ethics committee; all patients gave written consent before study participation after being thoroughly informed. Patients with verified CF were included if they were at least 18 years of age, were referred to clinic, and received unenhanced low-dose CT at the Department of Radiology (Charité University Medicine, Berlin, Germany) for clinical indications, such as acute pulmonary exacerbation. Besides $\mathrm{CT}$, all patients agreed to undergo an MR examination with the use of gadobutrol (Gd-DO3A-butriol; Gadovist, Bayer HealthCare Pharmaceuticals, Berlin, Germany), a macrocyclic contrast agent with low risk of developing nephrogenic systemic fibrosis. $^{25-27}$

Exclusion criteria were the following:

- Contraindications to MRI, eg, pacemaker, claustrophobia, severe obesity;

- Application of any contrast medium within 24 hours before the MRI examination with gadobutrol;

- History of anaphylactoid or anaphylactic reaction to any contrast medium;

- Pregnant or lactating women;

- Clinically unstable condition and/or requirement of emergency treatment and/or physical or mental status, which interfered with the signing of the informed consent;

- Chronic kidney disease $\geq 3$ (glomerular filtration rate $<60 \mathrm{~mL} \cdot \mathrm{min}^{-1}$ ) to further reduce the risk of developing nephrogenic systemic fibrosis.
The evaluation included CT and MR imaging as well as clinical, laboratory, and pulmonary functional examinations, which were all performed within 5 days to provide a reliable comparison between all parameters

\section{CT and MRI Protocols}

Chest CT was performed by a 64-slice multidetector system (GE Lightspeed VCT 64; General Electric Healthcare, Milwaukee, WI). A standardized low-dose unenhanced CT protocol was used with the following technical parameters in axial slice orientation and inspiratory breathhold: tube voltage, $100 \mathrm{kV}$, dose-modulated tube current, 35-80 mAs; pitch, 1.375; table speed per rotation, $55.0 \mathrm{~mm}$; reconstruction slice thickness, $0.625 \mathrm{~mm}$; and effective slice thickness, $5 \mathrm{~mm}$. Lung MRI was acquired using a 1.5-T system (Magnetom Avanto; Siemens Healthcare, Erlangen, Germany) with a standardized protocol. The technical parameters of the used T1- and T2-weighted MRI sequences are summarized in Table 1; gadobutrol was administered in a dose of $0.1-\mathrm{mmol} \cdot \mathrm{kg}^{-1}$ body weight.

\section{Analysis of Images}

All CT and MR images were visually evaluated in consensus by 2 radiologists with experience of at least 5 years in chest imaging. The observers evaluated all CT and MR images in a blinded manner to clinical and previous radiological data to achieve an appropriate comparison of imaging. The readers observed the images in a randomized patient order; to avoid recall bias, the time interval between the analysis of CT and MR examinations was at least 3 weeks. Two different scoring systems were used for all CT and MR images: the morphologic scoring system, developed by Helbich et $\mathrm{al}^{8}$ and modified by Puderbach et $\mathrm{al}^{12}$ for comparison between $\mathrm{CT}$ and MR examinations in patients with CF (Table 2) as well as the morphologic and functional scoring system developed by Eichinger et $\mathrm{al}^{22}$ for evaluation of MR images in patients with CF (Table 3).

For analysis of the specific CT and MR imaging parameters, the following descriptions were used according to published definitions:

- Bronchiectasis: ${ }^{12,22,28,29}$ was defined as bronchial dilatation with respect to the accompanying pulmonary artery, lack of tapering of bronchi, and identification of bronchi within $1 \mathrm{~cm}$ of the pleural surface; in the modified Helbich score, the severity of bronchiectasis

TABLE 1. Technical Parameters of the Sequences Used for the MRI of the Lung

\begin{tabular}{|c|c|c|c|c|}
\hline Parameters & HASTE & BLADE & VIBE & TWIST \\
\hline Repetition time, ms & 560 & 5500 & 3.7 & 2.4 \\
\hline Echo time, ms & 30 & 87 & 1.6 & 0.8 \\
\hline Flip angle, degree & 150 & 140 & 8 & 25 \\
\hline Acquisition matrix & 320 & 384 & 384 & 256 \\
\hline Field of view, mm & $400-500$ & $400-500$ & $400-500$ & $400-500$ \\
\hline GRAPPA & 2 & 2 & 2 & 3 \\
\hline Spatial resolution, $\mathrm{mm}^{3}$ & $1.3 \times 1.3 \times 5.0$ & $1.0 \times 1.0 \times 5.0$ & $1.0 \times 1.0 \times 5.0$ & $2.6 \times 1.6 \times 3.0$ \\
\hline Slice orientation & Axial & Axial & Axial & Coronal \\
\hline Breath-hold & $\begin{array}{l}\text { Yes; acquisition } \\
\text { time, } 16-19 \mathrm{~s}\end{array}$ & $\begin{array}{l}\text { No, respiratory navigator } \\
\text { triggering; mean acquisition } \\
\text { time, } 6.3 \text { minutes }\end{array}$ & $\begin{array}{l}\text { Yes; acquisition time per } \\
\text { breath-hold, 16-19 s }\end{array}$ & $\begin{array}{l}\text { No, free-breathing; } \\
25 \text { data sets with } \\
\text { acquisition time of } \\
\text { each, } 1.6 \mathrm{~s}\end{array}$ \\
\hline Contrast medium & $\begin{array}{l}\text { Acquisition before } \\
\text { the application of } \\
\text { contrast medium }\end{array}$ & $\begin{array}{l}\text { Acquisition before the } \\
\text { application of contrast } \\
\text { medium }\end{array}$ & $\begin{array}{l}\text { Acquisition of one sequence } \\
\text { before and one sequence } \\
\text { with the same technical } \\
\text { parameters after contrast } \\
\text { medium application }\end{array}$ & $\begin{array}{l}\text { Continuous acquisition } \\
\text { before, during, and } \\
\text { after application of } \\
\text { contrast medium }\end{array}$ \\
\hline
\end{tabular}

HASTE indicates half-Fourier acquisition single-shot turbo spin echo, T2-weighted; BLADE, proprietary name for a sequence using periodically rotated overlapping parallel lines with enhanced reconstruction, T2-weighted; VIBE, volumetric interpolated breath-hold examination, T1-weighted; TWIST, time-resolved angiography with stochastic trajectories, T1-weighted; GRAPPA, generalized autocalibrating partially parallel acquisition. 
TABLE 2. Modified Helbich Scoring System for Evaluation of CT and MR Imaging of the Lung*

\begin{tabular}{|c|c|c|c|c|}
\hline \multirow[b]{2}{*}{ Category } & \multicolumn{4}{|c|}{ Score } \\
\hline & $\mathbf{0}$ & 1 & 2 & 3 \\
\hline Severity of bronchiectasis $\dagger$ & Absent & $\begin{array}{l}\text { Mild (bronchial luminal } \\
\text { diameter slightly greater } \\
\text { than the diameter of the } \\
\text { adjacent blood vessel) }\end{array}$ & $\begin{array}{l}\text { Moderate (bronchial lumen } \\
2 \text { to } 3 \text { times greater than the } \\
\text { diameter of the adjacent } \\
\text { blood vessel) }\end{array}$ & $\begin{array}{l}\text { Severe (bronchial } \\
\text { lumen }>3 \text { times greater } \\
\text { than the diameter of the } \\
\text { adjacent blood vessel) }\end{array}$ \\
\hline $\begin{array}{l}\text { Severity of peribronchial } \\
\text { wall thickening } \dagger\end{array}$ & Absent & $\begin{array}{l}\text { Mild (wall thickness equal } \\
\text { to the diameter of the } \\
\text { adjacent vessel) }\end{array}$ & $\begin{array}{l}\text { Moderate (wall thickness } \\
\text { greater than and up to twice } \\
\text { the diameter of the } \\
\text { adjacent vessel) }\end{array}$ & $\begin{array}{l}\text { Severe (wall thickness } \\
\text { more than twice the } \\
\text { diameter of the } \\
\text { adjacent vessel) }\end{array}$ \\
\hline Extent of bronchiectasis $\ddagger$ & Absent & $1-5 \ddagger$ & $6-9 \ddagger$ & $>9 \ddagger$ \\
\hline Extent of mucus pluggingt: & Absent & $1-5 \ddagger$ & $6-9 \ddagger$ & $>9 \ddagger$ \\
\hline $\begin{array}{l}\text { Extent of sacculations/ } \\
\text { abscesses }\end{array}$ & Absent & $1-5 \ddagger$ & $6-9 \dagger$ & $>9 \$$ \\
\hline $\begin{array}{l}\text { Generation of bronchial } \\
\text { divisions involved } \\
\text { (bronchiectasis or } \\
\text { mucus plugging) }\end{array}$ & Absent & Up to the fourth generation & Up to the fifth generation & $\begin{array}{l}\text { Up to the sixth generation } \\
\text { and distal }\end{array}$ \\
\hline Severity of bullae & Absent & Unilateral (numbers not $>4$ ) & Bilateral (numbers not $>4$ ) & Numbers $>4$ \\
\hline Severity of emphysema & Absent & $1-5 \ddagger$ & $>5 t$ & Not applicable \\
\hline $\begin{array}{l}\text { Severity of collapse/ } \\
\text { consolidation }\end{array}$ & Absent & Subsegmental & Segmental or lobar & Not applicable \\
\hline
\end{tabular}

*According to the publication of Puderbach et al. ${ }^{12}$

$\dagger$ The severity of the parameters "bronchiectasis" and "peribronchial wall thickening" was recorded with that score (1-3), whereas most of the imaging features were present in the lung.

\$These categories are assessed on a segment-based analysis. The numbers represent the involved quantity of bronchopulmonary segments (right lung, 10 segments; left lung, 8 segments).

was estimated by comparing the bronchial luminal diameter with the size of the adjacent artery;

- Peribronchial wall thickening ${ }^{12,22}$ was separately assessed in the modified Helbich score in comparison with the adjacent artery; bronchiectasis and bronchial wall thickening were recorded as one single parameter in the Eichinger score;

- Mucus plugging ${ }^{12,21,22,28}$ was defined as fluid content within dilated bronchi greater than $3 \mathrm{~mm}$ without signal enhancement after contrast medium administration; peripheral mucus plugging can demonstrate a grapelike or "tree-in-bud" appearance. If mucus plugging was observed in the periphery, this segment or lobe was also scored for the parameter bronchiectasis;

- Sacculation/abscess ${ }^{12,22,29}$ was regarded as a circular structure with a diameter greater than $1.5 \mathrm{~cm}$, which was air-filled or showed an air-fluid level and had a thick wall;

- Collapse/consolidation ${ }^{12,22,28,29}$ was defined as a homogeneous increase in pulmonary parenchymal density (CT) or signal intensity (MRI) with a size greater than $2 \mathrm{~cm}$, which obscured the margins of vessels and airway walls; it can be caused by alveolar filling with inflammatory fluid and/or by atelectasis;

- Bulla ${ }^{12,28}$ was regarded as a sharply demarcated airspace with a diameter greater than $1 \mathrm{~cm}$ and a thin wall $(<1 \mathrm{~mm}$ thickness) in the modified Helbich score (Table 2). In the Eichinger score, this parameter was not considered;

- Emphysema ${ }^{12,28}$ was defined as an area of decreased density (CT) or signal intensity (MRI) compared with the surrounding lung parenchyma combined with a reduction of vessels. In the modified Helbich score, this parameter was documented on a segmental basis (Table 2). This imaging feature was not considered in the Eichinger score;

- Special findings ${ }^{22}$ : In the Eichinger score, pleural affection, eg, effusion or pneumothorax, was recorded as special finding (Table 3). This parameter was not documented in the modified Helbich score;
- Perfusion size ${ }^{22}$ : Eichinger et $\mathrm{al}^{22}$ described the size of a perfusion defect as the functional parameter perfusion size; the lung perfusion was assessed on the time point of maximum pulmonary enhancement on subtraction images of the time-resolved angiography with stochastic trajectories (TWIST) sequences (Tables 1 and 3). This

TABLE 3. Eichinger Scoring System for Evaluation of CT and MR Imaging of the Lung*

\begin{tabular}{|c|c|c|c|c|c|c|c|}
\hline \multirow[b]{2}{*}{ Parameter } & \multicolumn{3}{|c|}{ Right lung } & \multicolumn{3}{|c|}{ Left lung } & \multirow{2}{*}{$\begin{array}{c}\text { Maximal } \\
\text { Parameter/Globa } \\
\text { Score }\end{array}$} \\
\hline & UL & ML & $\mathbf{L L}$ & UL & LG & $\mathbf{L L}$ & \\
\hline $\begin{array}{l}\text { 1. Bronchiectasis/ } \\
\text { (peri-)bronchial wall } \\
\text { thickening } \dagger\end{array}$ & & & & & & & 12 \\
\hline 2. Mucus plugging $\dagger$ & & & & & & & 12 \\
\hline $\begin{array}{l}\text { 3. Sacculations/ } \\
\text { abscesses } \dagger\end{array}$ & & & & & & & 12 \\
\hline 4. Consolidation $\dagger$ & & & & & & & 12 \\
\hline 5. Special findings $\dagger$ & & & & & & & 12 \\
\hline 6. Perfusion size $\dagger t$ & & & & & & & 12 \\
\hline $\begin{array}{l}\text { Maximal lobar/ } \\
\text { global score }\end{array}$ & 12 & 12 & 12 & 12 & 12 & 12 & 72 \\
\hline
\end{tabular}

*According to the publication of Eichinger et al. ${ }^{22}$

$\dagger$ Each parameter is assessed on a lobe-based analysis by rating with 3 scores: 0 , pathology not present in this lobe; $1,<50 \%$ of the lobe involved; $2, \geq 50 \%$ of the lobe involved.

†The "perfusion size" is solely evaluated for the MR, not for the CT images. UL indicates upper lobe; ML, middle lobe; LL, lower lobe; LG, lingula. 
parameter was not determined in the evaluation of CT images and the analysis based on the modified Helbich score.

The modified Helbich score provided a maximum of 25 points (Table 2). The Eichinger score consisted of 2 subscores: morphologic score (parameters $1-5$, Table 3 ) with a maximum of 60 points (valid for CT and MRI) and functional score (lung perfusion only assessed on MRI, maximum of 12 points). In addition to the modified Helbich and Eichinger scores, the following MRI characteristics were analyzed: signal intensity of most of the peribronchial walls and visible mucus on T2-weighted images as well as the signal enhancement of most of peribronchial walls on contrast-enhanced volumetric interpolated breath-hold examination (VIBE) sequences (Table 1). For these evaluations, an ordinal 3-point scale was used:

- 0 = normal signal intensity and normal signal enhancement of the peribronchial walls/no mucus detected;

- 1 = moderately high signal intensity of the peribronchial walls and the mucus/moderately increased signal enhancement of the peribronchial walls;

- 2 = severely high signal intensity of the peribronchial walls and the mucus/severely increased signal enhancement of the peribronchial walls.

Furthermore, the readers assessed the subjective overall image quality of the CT and MR examinations on a 5-point Likert scale, where the reference was an "ideal exam": 30

-1 = nondiagnostic image quality;

- 2 = poor image quality, many artifacts;

- 3 = sufficient image quality for diagnostic purposes, some artifacts;

- 4 = good image quality, few artifacts;

- 5 = excellent image quality, no artifacts

\section{Clinical and Functional Characteristics}

The clinical status of the patients was determined by using the pulmonary exacerbation assessed by the modified criteria of Fuchs et al. ${ }^{31,32}$ According to these modified Fuchs criteria, ${ }^{31}$ pulmonary exacerbation was defined as the need for additional antibiotic treatment due to a recent change in at least 2 of the following 6 symptoms:

- Change in sputum volume and/or sputum color;

- Increased cough;

- Increased malaise, fatigue, or lethargy;

- Anorexia or significant weight loss;

- Decrease in pulmonary function by $10 \%$;

- Increased dyspnea.

For this study, the modified Fuchs criteria were used as a score ranging from 0 to 6 (based on the present clinical categories). As further clinical parameter, the presence of chronic pulmonary colonization by Pseudomonas aeruginosa of the patients was assessed. Serum levels of C-reactive protein in $\mathrm{mg} \cdot \mathrm{L}^{-1}$ and immunoglobulin $\mathrm{G}(\mathrm{IgG})$ in $\mathrm{mg} \cdot \mathrm{dL}^{-1}$, as well as the number of leucocytes per nanoliter $(\mathrm{nL})$ were recorded as blood laboratory values for inflammatory activity of $\mathrm{CF}$. Body plethysmography was performed as a pulmonary functional test and included the following parameters: forced vital capacity, forced expiratory volume in the first second, residual volume, effective resistance, and the maximum midexpiratory flow $25 \%$ to $75 \%$. The pulmonary functional results were expressed as percentages of reference values of healthy individuals.

\section{Statistical Evaluation}

Statistical analysis was performed using SPSS version 20.0 for Windows (IBM SPSS Statistics, Chicago, IL) and MedCalc version 14.0 for Windows (MedCalc Software; Mariakerke, Belgium). First, the prevalence of all clinical, pulmonary functional, and imaging parameters was determined. Differences between CT and MR examinations were assessed by Wilcoxon rank sum tests and Student $t$ tests. Concordance correlation coefficients (CCCs) were used to determine the agreement between CT and MR examinations for all imaging parameters. Spearman rank correlation coefficients were calculated to analyze the association of imaging findings to clinical findings including pulmonary functional results. Bland-Altman plots were performed for the overall modified Helbich and the Eichinger score to assess the overall agreement between CT and MRI; the mean of the score differences including the upper and the lower limits of agreement were determined. In all statistical tests, $P<0.05$ (2-sided) was considered to indicate significant differences.

\section{RESULTS}

\section{Patients' Characteristics}

Thirty-two patients with CF were enrolled in the study. Two patients had to be excluded of whom one patient refused the MR examination after initial written consent at the date of MR examination; the MR examination was interrupted in one patient owing to dyspnea during scanning. Thus, 30 patients [17 men and 13 women; mean (SD) age, 30.2 (9.2); range, 19-52 years] were included; their mean (SD) body mass index was $20.9(3.6) \mathrm{kg} \cdot \mathrm{m}^{-2}$; range, $14.6-32.1 \mathrm{~kg} \cdot \mathrm{m}^{-2}$. In the 30 patients, the following pulmonary exacerbation scores based on the modified Fuchs criteria were determined [mean (SD), 3.1 (2.1); median, 3.5; IQR, 4.0]: score $0, \mathrm{n}=5 ; 1, \mathrm{n}=5 ; 2, \mathrm{n}=2 ; 3, \mathrm{n}=3 ; 4, \mathrm{n}=4 ; 5$, $\mathrm{n}=8$; and $6, \mathrm{n}=3$. Twenty $(66.7 \%)$ of 30 patients had chronic pulmonary colonization by $P$. aeruginosa. The mean (SD) blood laboratory parameters of the 30 patients were as follows: C-reactive protein, 36.9 (54.4) $\mathrm{mg} \cdot \mathrm{L}^{-1}$ (range, $0.5-208.2 \mathrm{mg} \cdot \mathrm{L}^{-1}$ ); $\mathrm{IgG}, 1681.1(444.4) \mathrm{mg} \cdot \mathrm{dL}^{-1}$ (range, 941.0-2433.0 $\mathrm{mg} \cdot \mathrm{dL}^{-1}$ ), and number of leucocytes, 13.4 (4.9) per $\mathrm{nL}$ (range, 4.0-22.5 per $\mathrm{nL}$ ). The pulmonary functional tests resulted in the following mean (SD) values: forced vital capacity, $71.6 \%(23.2 \%)$ (range, 28.4\%-116.0\%); forced expiratory volume in the first second, $53.6 \%$ (22.9\%) (range, $21.0 \%-94.6 \%$ ); residual volume, $164.9 \%(56.7 \%)$ (range, 38.3\%-271.8\%); effective resistance, 214.2\% (118.6\%) (range, $57.1 \%-469.7 \%$ ); and maximum midexpiratory flow $25 \%$ to $75 \%, 27.6 \%$ $(22.5 \%$ ) (range, $8.9 \%-104.5 \%)$

\section{Imaging Findings}

Results of the imaging analysis by the modified Helbich score are summarized in Table 4 for the CT in comparison to the MR examinations. The differences of the scores between CT and MR examinations were not significant for all imaging features (Wilcoxon rank sum tests; $P>0.05)$. The overall modified Helbich CT score had a mean (SD) of 15.3 (4.8) (range, 3-21) and a median of 16.0 (IQR, 6.3). The overall modified Helbich MR score showed slightly, not significantly, lower values (Wilcoxon rank sum test and Student $t$ test; $P>0.05$ ): a mean (SD) of 14.3 (4.7) (range, 3-20) and a median of 15.0 (IQR, 7.3).

In accordance with the results of the modified Helbich score, all 30 patients showed bronchiectasis/peribronchial wall thickening on $\mathrm{CT}$ and MR examinations, assessed by the Eichinger score (Fig. 1). The mean and median scores of this imaging feature were as follows for CT versus MR examinations: mean (SD), 9.3 (2.4) (range, 4-12); median, 10.0 (IQR, 3.3) versus mean (SD), 9.0 (2.3) (range, 4-12); median, 9.0 (IQR, 3.0). Twenty-eight of the 30 patients had mucus plugging on $\mathrm{CT}$ and MR examinations with the following mean and median Eichinger scores: mean (SD), 7.9 (3.2) (range, 0-12); and median, 8.0 (IQR, 4.0) on CT; and mean (SD), 7.2 (3.3) (range, 0-12); and median, 7.5 (IQR, 5.0) on MRI (Fig. 2). In accordance with the modified Helbich score, one third of the patients (10/30 patients; $33.3 \%$ ) showed sacculations/abscesses on CT versus MRI with the following Eichinger scores: $1, \mathrm{n}=5 ; 2, \mathrm{n}=2 ; 4, \mathrm{n}=1 ; 6, \mathrm{n}=2$ versus 1 , $\mathrm{n}=5 ; 2, \mathrm{n}=3 ; 5, \mathrm{n}=1 ; 6, \mathrm{n}=1$. Consolidation was observed in 19 $(63.3 \%)$ of the 30 patients on CT: score $1, n=5 ; 2, n=6 ; 3, n=6 ; 4$, $\mathrm{n}=1 ; 6, \mathrm{n}=1$. On MRI, consolidation was found in $18(60.0 \%)$ of the 30 patients: score $1, n=4 ; 2, n=6 ; 3, n=5 ; 4, n=2 ; 6, n=1$. Special findings, that means pleural affection, were detected in $9(30.0 \%)$ of the 30 patients with the following Eichinger scores on CT: score 1, 
TABLE 4. Imaging Findings in 30 Patients With CF Assessed by the Modified Helbich Scoring System Based on CT and MR Examinations

\begin{tabular}{|c|c|c|c|c|c|c|c|c|}
\hline \multirow[b]{2}{*}{ Category } & \multicolumn{4}{|c|}{ Score Based on CT Examinations } & \multicolumn{4}{|c|}{ Score Based on MR Examinations } \\
\hline & $\mathbf{0}$ & 1 & 2 & 3 & $\mathbf{0}$ & 1 & 2 & 3 \\
\hline Severity of bronchiectasis & $0(0.0)$ & $5(16.7)$ & $11(36.7)$ & $14(46.7)$ & $0(0.0)$ & $6(20.0)$ & $10(33.3)$ & $14(46.7)$ \\
\hline Severity of peribronchial wall thickening & $3(10.0)$ & $9(30.0)$ & $10(33.3)$ & $8(26.7)$ & $5(16.7)$ & $7(23.3)$ & $13(43.3)$ & $5(16.7)$ \\
\hline Extent of bronchiectasis & $0(0.0)$ & $2(6.7)$ & $3(10.0)$ & $25(83.3)$ & $0(0.0)$ & $2(6.7)$ & $4(13.3)$ & $24(80.0)$ \\
\hline Extent of mucus plugging & $2(6.7)$ & $2(6.7)$ & $6(20.0)$ & $20(66.7)$ & $2(6.7)$ & $3(10.0)$ & $8(26.7)$ & $17(56.7)$ \\
\hline Extent of sacculations/abscesses & $20(66.7)$ & $7(23.3)$ & $1(3.3)$ & $2(6.7)$ & $20(66.7)$ & $8(26.7)$ & $1(3.3)$ & $1(3.3)$ \\
\hline $\begin{array}{l}\text { Generation of bronchial divisions involved } \\
\text { (bronchiectasis or mucus plugging) }\end{array}$ & $0(0.0)$ & $2(6.7)$ & $9(30.0)$ & $19(63.3)$ & $0(0.0)$ & $5(16.7)$ & $12(40.0)$ & $13(43.3)$ \\
\hline Severity of bullae & $14(46.7)$ & $5(16.7)$ & $4(13.3)$ & $7(23.3)$ & $15(50.0)$ & $5(16.7)$ & $4(13.3)$ & $6(20.0)$ \\
\hline Severity of emphysema & $12(40.0)$ & $8(26.7)$ & $10(33.3)$ & - & $16(53.3)$ & $6(20.0)$ & $8(26.7)$ & - \\
\hline Severity of collapse/consolidation & $11(36.7)$ & $11(36.7)$ & $8(26.7)$ & - & $12(40.0)$ & $10(33.3)$ & $8(26.7)$ & - \\
\hline
\end{tabular}

The data are presented as number of patients and percentages (in parentheses). The percentages may not add up to $100.0 \%$ owing to rounding off.

$\mathrm{n}=1 ; 2, \mathrm{n}=3 ; 3, \mathrm{n}=3 ; 4, \mathrm{n}=2$. On MRI, this imaging feature was observed in $12(40.0 \%)$ of the 30 patients: score $1, \mathrm{n}=3 ; 2, \mathrm{n}=4 ; 3, \mathrm{n}=2$; $4, \mathrm{n}=2 ; 5, \mathrm{n}=1$ (Fig. 2). Perfusion deficits were detected in $24(80.0 \%)$
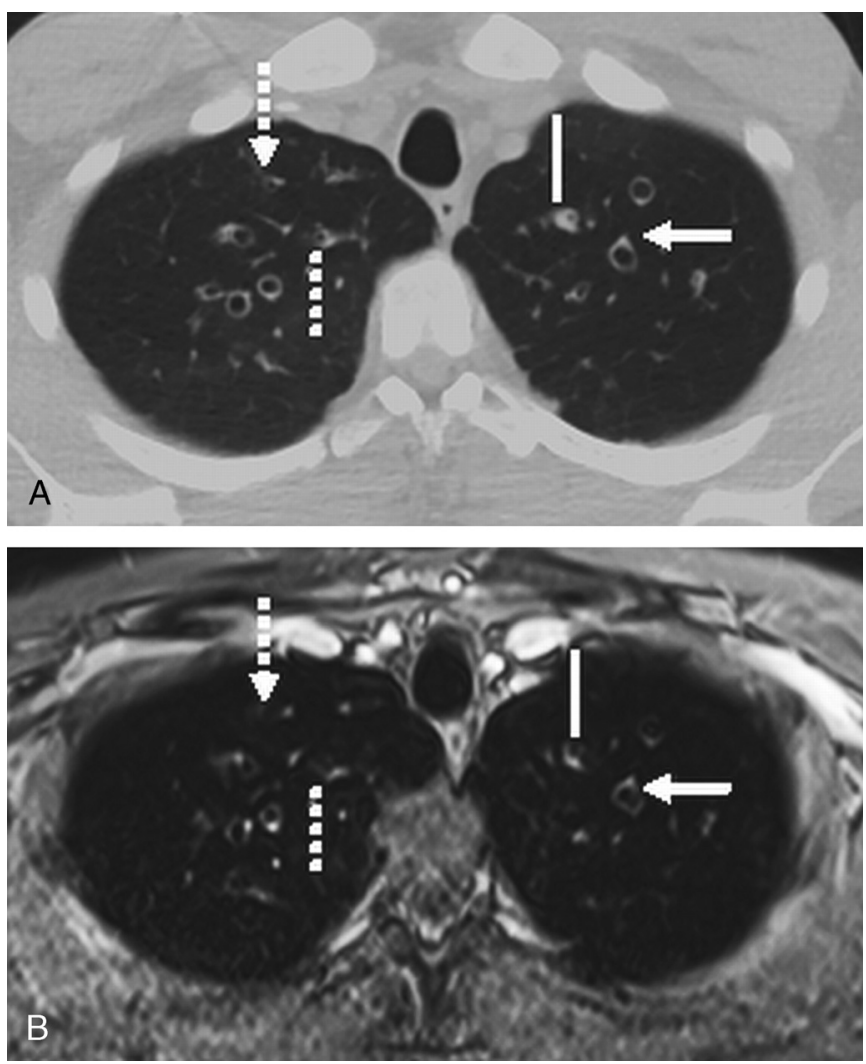

FIGURE 1. Computed tomography (A) and MR (B) examination of a 30-year-old male patient with CF. The unenhanced, low-dose, transversal CT image (A) shows bronchiectasis in the left and right upper lobe with peribronchial wall thickening, which is also visualized in the T1-weighted VIBE sequence (B), acquired after intravenous contrast medium administration (continuous lines). The VIBE sequence distinctly shows the characteristic signet ring sign of the bronchiectasis owing to the bright contrast enhancement of the accompanying bronchial arteries (continuous arrows). In the central lung area, small bronchiectasis with peribronchial wall thickening, clearly detected on $\mathrm{CT}$, can be also seen on MRI (discontinuous lines). In the lung periphery, small bronchiectasis can be visualized only on the $\mathrm{CT}$, not on the MR image (discontinuous arrows). of the 30 patients on MRI, with a mean (SD) score of 4.0 (3.2) and a median score of 3.5 (IQR, 6.3). Without assessment of perfusion, the overall Eichinger score resulted in the following values for CT versus MR examinations: mean (SD), 20.3 (7.2) (range, 4-31); and median,
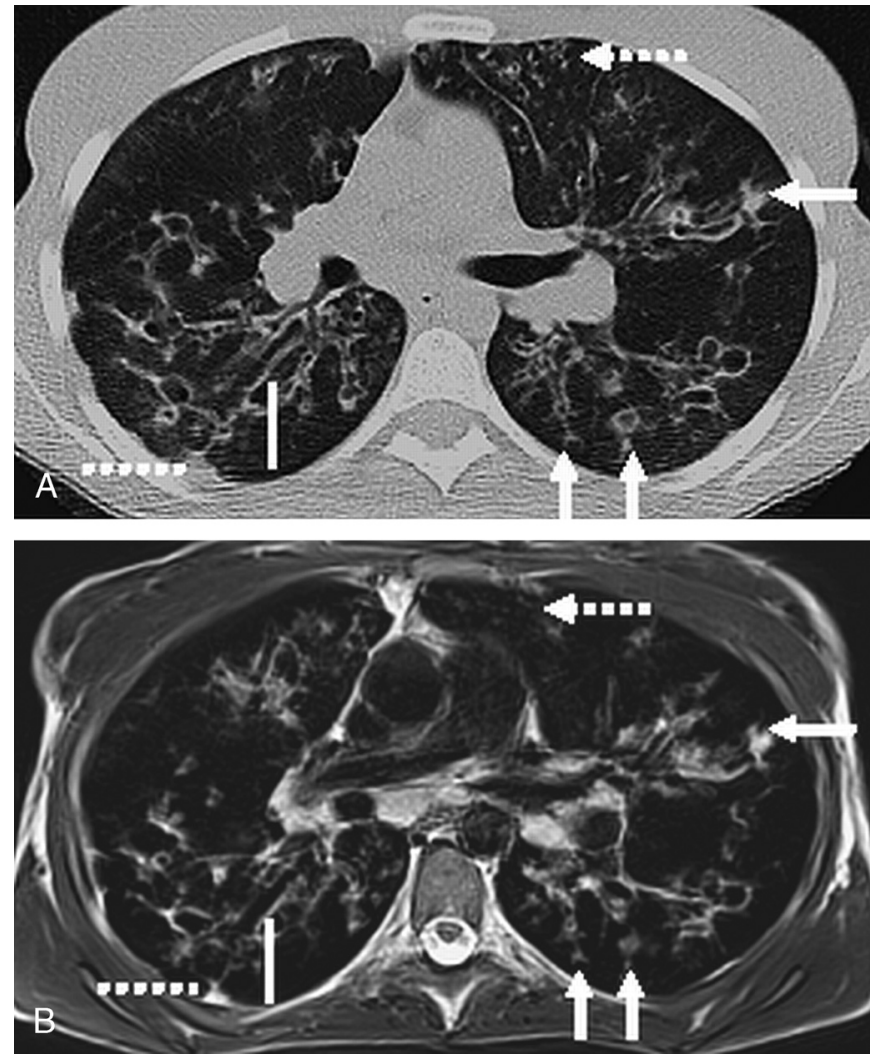

FIGURE 2. Computed tomography (A) and MR (B) examination of a 20-year-old female patient with CF. The unenhanced, low-dose, transversal CT image (A) demonstrates ubiquitary bronchiectasis with peribronchial wall thickening, which is also visualized in the T2-weighted BLADE sequence (B; continuous lines). Mucus plugging, detected clearly on CT, can be distinctly seen on MRI as well, even in the peripheral lung areas (continuous arrows). However, small bronchiectasis can be visualized only on the CT image, not on the MR image in the lung periphery (discontinuous arrows). The CT and the MR examinations show pleural affection in the right lung segment 6 (discontinuous lines). 
TABLE 5. Additional Imaging Characteristics in 30 Patients With CF Determined by MR Examinations

\begin{tabular}{lccc}
\hline Category & $\mathbf{0}$ & $\mathbf{1}$ & $\mathbf{2}$ \\
\hline $\begin{array}{l}\text { Contrast medium-induced signal } \\
\text { enhancement of the peribronchial walls }\end{array}$ & $8(26.7)$ & $11(36.7)$ & $11(36.7)$ \\
$\begin{array}{l}\text { T2-weighted signal intensity of the } \\
\text { peribronchial walls }\end{array}$ & $9(30.0)$ & $15(50.0)$ & $6(20.0)$ \\
$\begin{array}{l}\text { T2-weighted signal intensity } \\
\text { of detected mucus }\end{array}$ & $2(6.7)$ & $13(43.3)$ & $15(50.0)$ \\
\hline
\end{tabular}

The data are presented as number of patients and percentages (in parentheses). The percentages may not add up to $100.0 \%$ owing to rounding off.

21.0 (IQR, 9.5) versus mean (SD), 19.5 (7.1) (range, 4-33); and median, 20.0 (IQR, 9.0). All differences between CT and MR examinations were not significant (Wilcoxon rank sum tests and Student $t$ tests; $P>0.05$ ).

The prevalences of the additional MRI characteristic signal intensity/signal enhancement of peribronchial walls and signal intensity of detected mucus are presented in Table 5. The overall image quality was higher for the CT examinations compared to the MRI sequences: mean (SD), 4.1 (0.8) (range, 3-5); median, 4.0 (IQR, 1.3) versus mean (SD), 3.5 (0.8) (range, 2-5); median, 3.5 (IQR, 1.0); the difference of image quality reached statistical significance (Wilcoxon rank sum test and Student $t$ test; $P<0.05$ ).

\section{Associations Between Imaging and Clinical Findings}

Using the modified Helbich score, the CCCs between the different $\mathrm{CT}$ and MR imaging parameters ranged from 0.57 (category generation of bronchial divisions involved) to 0.97 (parameter severity of collapse/consolidation); the CCC was 0.94 for the overall scoring system. For the Eichinger score, the CCCs between the different CT and MR imaging parameters ranged from 0.84 (category special findings) to 0.98 (parameter consolidation). The overall Eichinger score (without perfusion measurement) showed a CCC of 0.95 between CT and MR examinations.

The CT and MRI values from both scoring systems showed no difference in patients with chronic pulmonary colonization by $P$. aeruginosa compared to those without chronic pulmonary colonization (Wilcoxon rank sum tests and Student $t$ tests; $P>0.05$ ). Correlation coefficients between imaging findings in comparison with the pulmonary exacerbation rate score (modified Fuchs criteria), blood laboratory results, and pulmonary functional test are summarized in Tables 6 and 7 . In general, these correlations were higher for both overall scoring systems compared with the specific imaging parameters. The correlations of the CT scores to the clinical, laboratory, and pulmonary functional parameters were slightly higher compared with the MRI scores (Tables 6 and 7). However, if the additional MRI parameters were integrated into the scoring systems, the correlations reached the values of the CT scores (Tables 6 and 7). The results of the Bland-Altman plots are shown in Figure 3 (for the overall modified Helbich score) and Figure 4 (for the overall Eichinger score); the Bland-Altman plots revealed a strong agreement between MR and CT imaging for both scores

TABLE 6. Spearman Correlation Coefficients Between CT and MR Imaging Results (Using the Modified Helbich Scoring System), Clinical Findings, and Pulmonary Functional Test Results in 30 Patients With CF

\begin{tabular}{|c|c|c|c|c|c|c|c|c|c|}
\hline Parameters & $\begin{array}{l}\text { Score of Modified } \\
\text { Fuchs criteria }\end{array}$ & CRP & IgG & No. of leucocytes & FVC & FEV1 & RV & $\mathbf{R}$ & MMEF 75/25 \\
\hline CT: Severity of bronchiectasis & $0.75 \dagger$ & $0.42 *$ & 0.19 & $0.42 *$ & $-0.60 \dagger$ & $-0.62 \dagger$ & $0.57 \dagger$ & $0.44^{*}$ & $-0.59 \dagger$ \\
\hline MRI: Severity of bronchiectasis & $0.67 \dagger$ & $0.43^{*}$ & $0.37^{*}$ & $0.39 *$ & $-0.54 \dagger$ & $-0.59 \dagger$ & $0.51 \dagger$ & $0.46^{*}$ & $-0.57 \dagger$ \\
\hline CT: Severity of peribronchial wall thickening & $0.50 \dagger$ & $0.43^{*}$ & 0.28 & $0.52 \dagger$ & $-0.45^{*}$ & $-0.47 *$ & $0.37 *$ & $0.38^{*}$ & $-0.49 *$ \\
\hline MRI: Severity of peribronchial wall thickening & $0.46^{*}$ & $0.41^{*}$ & 0.18 & $0.53 \dagger$ & $-0.41^{*}$ & $-0.47^{*}$ & 0.30 & $0.38^{*}$ & $-0.47^{*}$ \\
\hline CT: Extent of bronchiectasis & $0.51 \dagger$ & $0.37 *$ & 0.12 & $0.44 *$ & $-0.31^{*}$ & $-0.43 *$ & $0.62 \dagger$ & $0.45^{*}$ & $-0.51 \dagger$ \\
\hline MRI: Extent of bronchiectasis & $0.46^{*}$ & $0.33^{*}$ & 0.09 & $0.40 *$ & -0.29 & $-0.42 *$ & $0.59 \dagger$ & $0.42 *$ & $-0.49^{*}$ \\
\hline CT: Extent of mucus plugging & $0.58 \dagger$ & $0.61 \dagger$ & 0.21 & $0.49 *$ & $-0.38^{*}$ & $-0.49 *$ & $0.47 *$ & $0.45^{*}$ & $-0.49 *$ \\
\hline MRI: Extent of mucus plugging & $0.54 \dagger$ & $0.62 \dagger$ & 0.23 & $0.43 *$ & $-0.36^{*}$ & $-0.48^{*}$ & $0.43 *$ & $0.43 *$ & $-0.48^{*}$ \\
\hline CT: Extent of sacculations/abscesses & $0.45^{*}$ & 0.27 & 0.19 & 0.27 & $-0.42^{*}$ & $-0.47 *$ & $0.44 *$ & $0.43^{*}$ & $-0.53 \dagger$ \\
\hline MRI: Extent of sacculations/abscesses & $0.44^{*}$ & 0.29 & 0.17 & 0.27 & $-0.40^{*}$ & $-0.45^{*}$ & $0.43 *$ & $0.41^{*}$ & $-0.51 \dagger$ \\
\hline CT: Generation of bronchial divisions involved & $0.47 *$ & $0.43 *$ & $0.44^{*}$ & $0.51 \dagger$ & $-0.35^{*}$ & $-0.50 \dagger$ & $0.53 \dagger$ & $0.46^{*}$ & $-0.51 \dagger$ \\
\hline MRI: Generation of bronchial divisions involved & $0.36^{*}$ & $0.41 *$ & 0.28 & $0.42 *$ & -0.29 & $-0.35^{*}$ & $0.39 *$ & $0.31 *$ & $-0.37 *$ \\
\hline CT: Severity of bullae & 0.27 & 0.28 & 0.06 & 0.14 & -0.18 & -0.27 & $0.41 *$ & 0.24 & -0.20 \\
\hline MRI: Severity of bullae & 0.25 & 0.26 & 0.07 & 0.21 & -0.19 & -0.26 & $0.35 *$ & 0.24 & -0.18 \\
\hline CT: Severity of emphysema & 0.26 & 0.16 & 0.10 & $0.39 *$ & -0.26 & $-0.34 *$ & $0.38 *$ & $0.36^{*}$ & $-0.45^{*}$ \\
\hline MRI: Severity of emphysema & 0.18 & 0.09 & 0.06 & $0.34 *$ & -0.23 & $-0.31^{*}$ & $0.36^{*}$ & 0.27 & $-0.41 *$ \\
\hline CT: Severity of collapse/consolidation & 0.24 & 0.21 & 0.22 & $0.38 *$ & $-0.36^{*}$ & $-0.30^{*}$ & 0.22 & 0.22 & -0.27 \\
\hline MRI: Severity of collapse/consolidation & 0.21 & 0.22 & 0.18 & $0.32 *$ & $-0.33^{*}$ & -0.27 & 0.18 & 0.18 & -0.26 \\
\hline Overall modified Helbich CT score & $0.67 \dagger$ & $0.62 \dagger$ & $0.32^{*}$ & $0.63 \dagger$ & $-0.53 \dagger$ & $-0.63 \dagger$ & $0.66 \dagger$ & $0.54 \dagger$ & $-0.65 \dagger$ \\
\hline Overall modified Helbich MRI score & $0.58 \dagger$ & $0.57 \dagger$ & 0.26 & $0.59 \dagger$ & $-0.51 \dagger$ & $-0.59 \dagger$ & $0.58 \dagger$ & $0.51 \dagger$ & $-0.62 \dagger$ \\
\hline $\begin{array}{l}\text { Overall modified Helbich MRI score } \\
\text { plus additional } 3 \text { MRI parameters }\end{array}$ & $0.69 \dagger$ & $0.63 \dagger$ & 0.28 & $0.66 \dagger$ & $-0.54 \dagger$ & $-0.61 \dagger$ & $0.63 \dagger$ & $0.53 \dagger$ & $-0.64 \dagger$ \\
\hline
\end{tabular}

$* P<0.05$

$\dagger P<0.01$.

CRP indicates C-reactive protein; IgG, immunoglobulin G; FVC, forced vital capacity; FEV1, forced expiratory volume in the first second; RV, residual volume; R, effective resistance; MMEF 75/25, maximum midexpiratory flow $25 \%-75 \%$. 
TABLE 7. Spearman Correlation Coefficients Between CT and MR Imaging Results (Using the Eichinger Scoring System), Clinical Findings, and Pulmonary Functional Test Results in 30 Patients with CF

\begin{tabular}{|c|c|c|c|c|c|c|c|c|c|}
\hline Parameters & $\begin{array}{l}\text { Score of Modified } \\
\text { Fuchs Criteria }\end{array}$ & CRP & IgG & No. of leucocytes & FVC & FEV1 & $\mathbf{R V}$ & $\mathbf{R}$ & MMEF 75/25 \\
\hline CT: Bronchiectasis/peribronchial wall thickening & $0.58 \dagger$ & $0.68 \dagger$ & $0.42 *$ & $0.47 *$ & $-0.46^{*}$ & $-0.58 \dagger$ & $0.60 \dagger$ & $0.49 *$ & $-0.65 \dagger$ \\
\hline MRI: Bronchiectasis/peribronchial wall thickening & $0.59 \dagger$ & $0.64 \dagger$ & $0.33^{*}$ & $0.48^{*}$ & $-0.42 *$ & $-0.55 \dagger$ & $0.57 \dagger$ & $0.48^{*}$ & $-0.60 \dagger$ \\
\hline CT: Mucus plugging & $0.66 \dagger$ & $0.61 \dagger$ & $0.40^{*}$ & $0.58 \dagger$ & $-0.64 \dagger$ & $-0.68 \dagger$ & $0.61 \dagger$ & $0.58 \dagger$ & $-0.70 \dagger$ \\
\hline MRI: Mucus plugging & $0.60 \dagger$ & $0.55 \dagger$ & $0.31^{*}$ & $0.55 \dagger$ & $-0.58 \dagger$ & $-0.64 \dagger$ & $0.45^{*}$ & $0.56 \dagger$ & $-0.61 \dagger$ \\
\hline CT: Sacculations/abscesses & $0.46^{*}$ & 0.24 & 0.18 & 0.28 & $-0.43^{*}$ & $-0.47 *$ & $0.47 *$ & $0.42 *$ & $-0.53 \dagger$ \\
\hline MRI: Sacculations/abscesses & $0.44 *$ & 0.25 & 0.18 & 0.28 & $-0.41^{*}$ & $-0.46^{*}$ & $0.46^{*}$ & $0.41^{*}$ & $-0.52 \dagger$ \\
\hline CT: Consolidation & 0.24 & 0.19 & $0.37^{*}$ & $0.35^{*}$ & $-0.37^{*}$ & $-0.30 *$ & 0.17 & 0.21 & -0.26 \\
\hline MRI: Consolidation & 0.22 & 0.18 & $0.38^{*}$ & $0.33^{*}$ & $-0.35^{*}$ & -0.29 & 0.12 & 0.19 & -0.23 \\
\hline CT: Special findings & 0.23 & 0.29 & 0.24 & $0.33^{*}$ & $-0.42 *$ & $-0.45^{*}$ & 0.29 & $0.40^{*}$ & $-0.44 *$ \\
\hline MRI: Special findings & 0.18 & 0.25 & 0.17 & 0.27 & $-0.41^{*}$ & $-0.40^{*}$ & 0.24 & $0.35^{*}$ & $-0.40 *$ \\
\hline Overall Eichinger CT score without perfusion & $0.68 \dagger$ & $0.63 \dagger$ & $0.41^{*}$ & $0.62 \dagger$ & $-0.71 \dagger$ & $-0.72 \dagger$ & $0.64 \dagger$ & $0.64 \dagger$ & $-0.73 \dagger$ \\
\hline Overall Eichinger MRI score without perfusion & $0.65 \dagger$ & $0.55 \dagger$ & $0.39^{*}$ & $0.60 \dagger$ & $-0.64 \dagger$ & $-0.67 \dagger$ & $0.57 \dagger$ & $0.62 \dagger$ & $-0.70 \dagger$ \\
\hline MRI: Perfusion size & $0.61 \dagger$ & $0.62 \dagger$ & $0.40^{*}$ & $0.57 \dagger$ & $-0.58 \dagger$ & $-0.65 \dagger$ & $0.51 \dagger$ & $0.53 \dagger$ & $-0.64 \dagger$ \\
\hline Overall Eichinger MRI score with perfusion & $0.67 \dagger$ & $0.69 \dagger$ & $0.44^{*}$ & $0.61 \dagger$ & $-0.66 \dagger$ & $-0.71 \dagger$ & $0.58 \dagger$ & $0.63 \dagger$ & $-0.73 \dagger$ \\
\hline $\begin{array}{l}\text { Overall Eichinger MRI score with perfusion } \\
\text { plus additional } 3 \text { MRI parameters }\end{array}$ & $0.71 \dagger$ & $0.70 \dagger$ & $0.47^{*}$ & $0.66 \dagger$ & $-0.67 \dagger$ & $-0.71 \dagger$ & $0.60 \dagger$ & $0.65 \dagger$ & $-0.74 \dagger$ \\
\hline
\end{tabular}

$* P<0.05$.

$\dagger P<0.01$.

CRP indicates C-reactive protein; IgG, immunoglobulin G; FVC, forced vital capacity; FEV1, forced expiratory volume in the first second; RV, residual volume; $\mathrm{R}$, effective resistance; MMEF 75/25, maximum midexpiratory flow $25 \%-75 \%$.

(means of the difference: overall modified Helbich score, 1.1; and overall Eichinger score, 0.8).

\section{DISCUSSION}

Supported by continuously improved technical developments, MRI has increasingly been discussed as an adequate radiation-free alternative to CT for some lung disorders. In this study, we could verify that if all information from the MR images is considered, MRI can demonstrate similar correlation to laboratory and pulmonary functional parameters as well as to the grade of pulmonary exacerbation at the time of lung imaging. Additionally, the concordance between CT and MR examinations was very high, with coefficients of 0.94 (overall modified Helbich score) and 0.95 (overall Eichinger score without perfusion measurement). Puderbach et $\mathrm{al}^{12}$ also performed a comparison study of CT

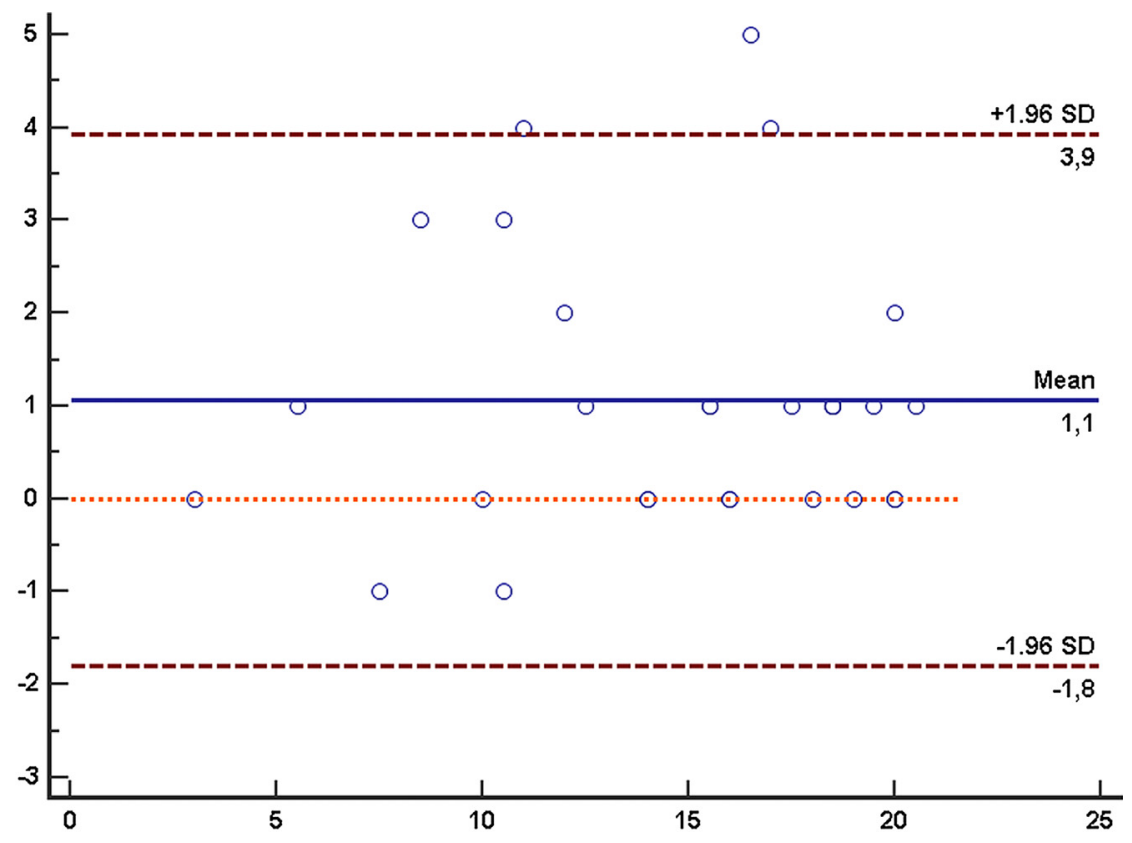

FIGURE 3. The Bland-Altman plot shows the difference of the overall modified Helbich score between the CT and MR examinations in 30 patients with CF (the image only shows 23 points because of the overlap of 7 values). With a probability of $95 \%$, the differences between further CT and MR examinations will be in-between the upper limit of agreement (+3.9) and the lower limit of agreement $(-1.8)$. Figure 3 can be viewed online in color at www.investigativeradiology.com. 


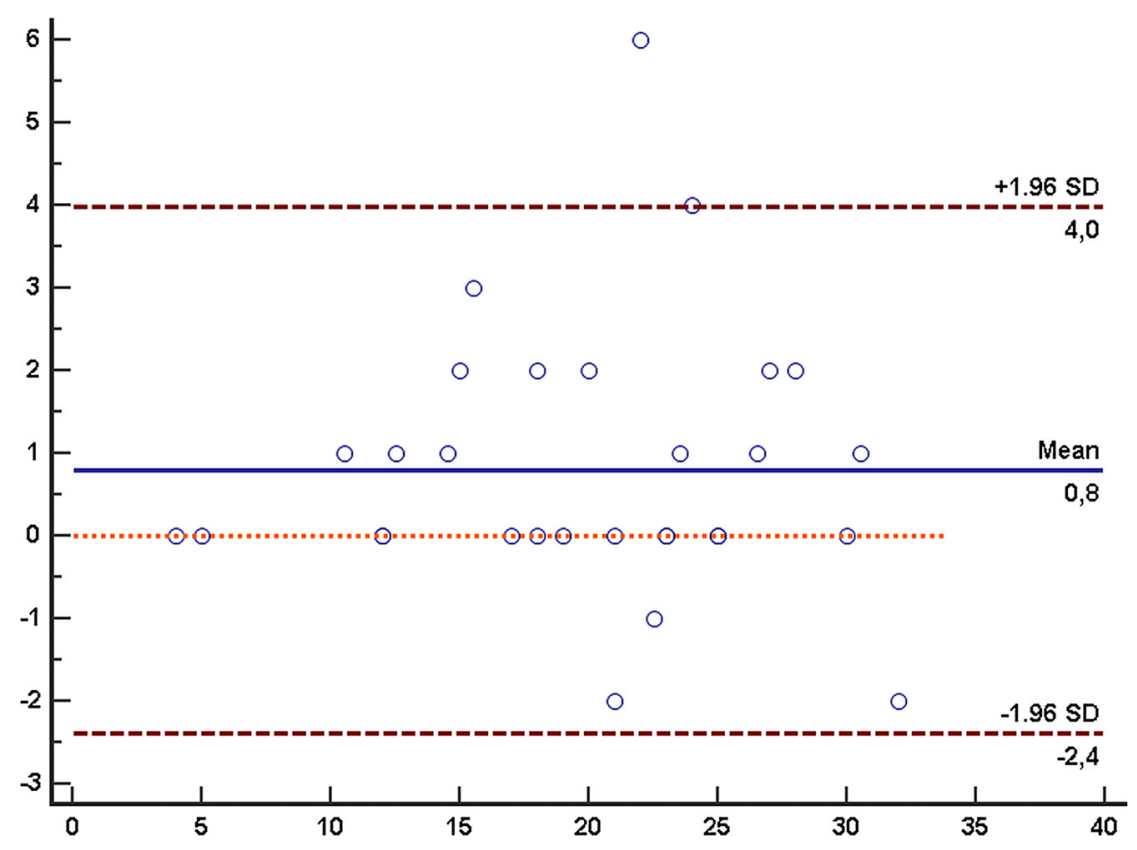

FIGURE 4. The Bland-Altman plot shows the difference of the overall Eichinger score between the CT and MR examinations in 30 patients with CF (the image only shows 27 points because of the overlap of 3 values). With a probability of $95 \%$, the differences between further CT and MR examinations will be in-between the upper limit of agreement $(+4.0)$ and the lower limit of agreement $(-2.4)$. Figure 4 can be viewed online in color at www.investigativeradiology.com.

and MRI in 31 patients with CF using the modified Helbich score without, however, considering any clinical or additional MRI parameters. The authors found an overall correlation of 0.80 between CT and MR images. ${ }^{12}$ Montella et $\mathrm{al}^{33}$ investigated whether 3-T MRI was as effective as CT in identifying pulmonary abnormalities in primary ciliary dyskinesia also using the modified Helbich score; the overall correlation of 0.95 in this study was similar to our findings. One possible explanation for the inferior correlation in the study of Puderbach et $\mathrm{al}^{12}$ could be that the patients were younger (mean age, 16.7 years), resulting in a lower median overall score of 13.0 (MRI) and 13.5 (CT).

Despite technical developments, subtle pulmonary changes and peripheral bronchi (resulting in a relatively low correlation of the category generation of bronchial divisions involved between MRI and CT) can be still less clearly visualized on MRI owing to its lower spatial resolution. $^{12,21}$ Therefore, the CT examinations reached in general higher values in all morphologic parameters of both scoring systems in our study. Owing to breathing artifacts in breath-hold MRI sequences, the impaired spatial resolution, and the relatively low and heterogeneous pulmonary signal-to-noise ratio, the diagnostic image quality of lung MRI is challenging. ${ }^{12,21,34}$ In this consequence, the overall diagnostic image quality was significantly inferior for MR compared with CT examinations in our investigation. However, all MR examinations were of diagnostic quality with a mean value of 3.5 (between sufficient and good image quality); in 3 patients with $\mathrm{CF}$, the MR examination was rated as score 2 (poor image quality with many artifacts). All 3 patients had severe CF lung manifestation with decreased pulmonary functional capacity and therefore CF-related limitations in sufficient breath-hold, leading to poor image quality in particularly the half-Fourier acquisition single-shot turbo spin echo (HASTE) and volumetric interpolated breath-hold examination (VIBE) sequences. Thus, sequences acquired with respiratory navigating should be part of a routine protocol of lung MRI, especially in patients with impaired clinical status.

It has been not evaluated in detail so far, whether the clear visualization of subtle pulmonary pathologies are important for the severity assessment and the prognosis of CF lung disease. Thus, we correlated CT and MR imaging findings to the clinical status of the patients including their pulmonary exacerbation grade. Helbich et $\mathrm{al}^{8}$ compared CT imaging (assessed by the Helbich score) with pulmonary functional test results, serum IgG values, and a clinical scoring system in patients with CF. The Spearman correlation coefficients between the different morphologic CT parameters and the $\mathrm{IgG}$ levels ranged from 0.29 to 0.69 in the study of Helbich et $\mathrm{al}^{8}{ }^{8}$ indicating higher values than our correlations with IgG levels (CT, 0.06-0.44). Hypergammaglobulinemia has been proven to predict pulmonary progression in $\mathrm{CF}^{35}$; however, only few patients with $\mathrm{CF}$ demonstrate manifest hypergammaglobulinemia, minimizing its importance for the determination of CF disease severity. ${ }^{35,36}$

The Spearman correlation coefficients between CT imaging and pulmonary functional parameters, published by Helbich et $\mathrm{al}^{8}{ }^{8}$ were similar to our calculated coefficients. Accordingly, some imaging features showed higher correlations to pulmonary functional parameters, such as severity/extent of bronchiectasis or extent of mucus plugging, than others, such as severity of bullaelemphysema; whereas these differences were similar for CT and MRI. Computed tomography scores have been described to can be even more sensitive than pulmonary functional parameters in the detection of early CF lung disease and in determining the progression of lung manifestation. ${ }^{10,29,37,38}$ Oikonomou et al ${ }^{10}$ reported that simplification of the CT scoring systems provided even better findings than the original more extent scores. The more simplified structure of the Eichinger scoring system might be one main reason that the Eichinger score showed in general higher clinical correlations and stronger agreement between CT and MRI demonstrated in the Bland-Altman plot (Fig. 4) than the modified Helbich scoring system. Furthermore, the Eichinger score was specifically developed for the assessment of lung MRI in CF. Wielpütz et $\mathrm{al}^{14}$ also used the Eichinger scoring system for the assessment of lung MRI in children with early CF lung disease; in patients with pulmonary exacerbations, the overall mean score was 18.0 (SD 2.0) and was significantly reduced to 12.0 (SD 3.0) after antibiotic treatment. ${ }^{14}$

Some imaging features have been omitted in the Eichinger scoring system, such as the separate analysis of severity of bronchiectasis and severity of peribronchial wall thickening. The parameter bronchiectasis has been proven as an outcome predictor for the number of 
pulmonary exacerbations in CF over a 2-year period. ${ }^{7}$ Peribronchial wall thickening might represent more distinctly the inflammatory activity of the peribronchial walls at the time of pulmonary exacerbation; high T2-weighted signal (ie, edema) and strong contrast medium enhancement of the peribronchial walls might reflect the inflammatory activity even more specifically. ${ }^{13,21,29}$ As far as we know, the structured, detailed MRI evaluation of the peribronchial walls and of visible mucus was performed in this study for the first time in CF.

Another parameter, which could be solely assessed on MRI, is the functional parameter of lung perfusion. On CT, this parameter can be determined only if a relatively high additional $\mathrm{x}$-ray exposure is used. Despite the upcoming possibility of potent iterative reconstruction techniques to reduce radiation exposure, the cumulative radiation exposure of CT examinations can still become complicated for patients with CF, particularly if additional series, such as lung perfusion, are acquired. ${ }^{8-11,31,39}$ Pulmonary perfusion CT can be assessed using dualenergy technique with less but also additional radiation dose. ${ }^{40}$ The correlation of MR perfusion imaging to clinical and especially pulmonary functional parameters was high in our study, as both assessments are of functional origin. According to Wielpütz et al, ${ }^{14}$ regional perfusion deficits are early signs of pulmonary manifestations in CF and can be even detected in infants and preschool children in stable clinical condition. Lung perfusion imaging has a relatively low acquisition time of up to 2 minutes and should therefore be part of a routine MRI protocol in $\mathrm{CF}$. When all additional MRI parameters were considered, the correlations between MR imaging and clinical, laboratory, and pulmonary functional parameters were similar to the correlation coefficients of CT examinations, although the overall image quality of MRI was significantly inferior. Thus, the imaging of subtle pulmonary pathologies by CT seems to be of minor clinical importance.

The following limitations of this study should be noted. All images were evaluated in consensus, and therefore inter-reader variability was not calculated; Eichinger et $\mathrm{al}^{22}$ reported a high inter-reader agreement (CCC 0.97) of the overall MR Eichinger score. As the inclusion criteria necessitated a homogeneous study design, such as data acquisition within 5 days, only a limited number of 30 patients could be included. The time interval of maximum 5 days between the 2 imaging examinations and the acquisition of clinical, laboratory, and pulmonary functional parameters might lead to some disproportionate correlations. However, in the trial of Puderbach et al, ${ }^{12}$ the time interval between CT and MRI was at maximum 4 weeks; this higher interval might be one reason that the agreement between the 2 imaging modalities was lower than in our study: mean of the difference in the Bland-Altman plot of the modified Helbich score, 2.7 versus 1.1 (Fig. 3). Another reason for the higher agreement in our study might be that the median overall modified Helbich score of CT and MRI was higher in our investigation compared to the values reported by Puderbach et al. ${ }^{12}$ Thus, the older patients of our study demonstrated more severe imaging pathologies, which might provide better correlations of MR to CT examinations as well as clinical, laboratory, and functional parameters. Helbich et $\mathrm{al}^{8}$ found a significantly higher onset of all CT parameters with increasing age of the patients with CF. A distinct evaluation of the MRI scoring systems in the case of early pulmonary CF changes especially in children, including a detailed comparison to their clinical status, would further advance the implementation of lung MRI in patients with CF in a clinical setting.

\section{CONCLUSIONS}

The findings of this prospective investigation indicate that MRI of the lung provide similar correlations to clinical, laboratory, and pulmonary functional parameters compared with chest CT, even if the overall image quality of MRI is inferior. Thus, lung MRI can also be used as a reliable surrogate marker for the severity of pulmonary manifestation in patients with $\mathrm{CF}$, as an alternative to chest CT. A principal advantage of MRI is the possibility to determine multiple morphologic and functional pulmonary imaging features without the use of any radiation exposure, particularly with regard to the continuously increasing life expectancy of patients with CF.

\section{ACKNOWLEDGMENT}

The authors thank Virginia Ding-Reinelt and Carina Schuecke (both from Berlin, Germany) for expert technical assistance.

\section{REFERENCES}

1. Goss CH, Ratjen F. Update in cystic fibrosis 2012. Am J Respir Crit Care Med. 2013; 187:915-919.

2. Ng MY, Flight W, Smith E. Pulmonary complications of cystic fibrosis. Clin Radiol. 2014;69:e153-e162.

3. Gibson RL, Burns JL, Ramsey BW. Pathophysiology and management of pulmonary infections in cystic fibrosis. Am J Respir Crit Care Med. 2003;168:918-951.

4. Dodge JA, Lewis PA, Stanton M, et al. Cystic fibrosis mortality and survival in the UK: 1947-2003. Eur Respir J. 2007;29:522-526.

5. Stern M, Wiedemann B, Wenzlaff P. From registry to quality management: the German Cystic Fibrosis Quality Assessment project 1995-2006. Eur Respir J. 2008;31:29-35.

6. Davis SD, Fordham LA, Brody AS, et al. Computed tomography reflects lower airway inflammation and tracks changes in early cystic fibrosis. Am J Respir Crit Care Med. 2007; 175:943-950.

7. Brody AS, Sucharew H, Campbell JD, et al. Computed tomography correlates with pulmonary exacerbations in children with cystic fibrosis. Am J Respir Crit Care Med. 2005;172:1128-1132.

8. Helbich TH, Heinz-Peer G, Eichler I, et al. Cystic fibrosis: CT assessment of lung involvement in children and adults. Radiology. 1999;213:537-544.

9. de Jong PA, Tiddens HA. Cystic fibrosis specific computed tomography scoring. Proc Am Thorac Soc. 2007;4:338-342.

10. Oikonomou A, Tsanakas J, Hatziagorou E, et al. High resolution computed tomography of the chest in cystic fibrosis (CF): is simplification of scoring systems feasible? Eur Radiol. 2008;18:538-547.

11. Fazel R, Krumholz HM, Wang Y, et al. Exposure to low-dose ionizing radiation from medical imaging procedures. N Engl J Med. 2009;361:849-857.

12. Puderbach M, Eichinger M, Haeselbarth J, et al. Assessment of morphological MRI for pulmonary changes in cystic fibrosis (CF) patients: comparison to thinsection CT and chest x-ray. Invest Radiol. 2007;42:715-725.

13. Wielpütz MO, Eichinger M, Puderbach M. Magnetic resonance imaging of cystic fibrosis lung disease. J Thorac Imaging. 2013;28:151-159.

14. Wielpütz MO, Puderbach M, Kopp-Schneider A, et al. Magnetic resonance imaging detects changes in structure and perfusion, and response to therapy in early cystic fibrosis lung disease. Am J Respir Crit Care Med. 2014;189:956-965.

15. Fiel SB, Friedman AC, Caroline DF, et al. Magnetic resonance imaging in young adults with cystic fibrosis. Chest. 1987;91:181-184.

16. Carr DH, Oades P, Trotman-Dickenson B, et al. Magnetic resonance scanning in cystic fibrosis: comparison with computed tomography. Clin Radiol. 1995; 50:84-89.

17. Montella S, Santamaria F, Salvatore M, et al. Assessment of chest high-field magnetic resonance imaging in children and young adults with noncystic fibrosis chronic lung disease: comparison to high-resolution computed tomography and correlation with pulmonary function. Invest Radiol. 2009;44:532-538.

18. Stolzmann P, Veit-Haibach P, Chuck N, et al. Detection rate, location, and size of pulmonary nodules in trimodality PET/CT-MR: comparison of low-dose CT and Dixon-based MR imaging. Invest Radiol. 2013;48:241-246.

19. Wurnig MC, Tsushima Y, Weiger M, et al. Assessing lung transplantation ischemia-reperfusion injury by microcomputed tomography and ultrashort echo-time magnetic resonance imaging in a mouse model. Invest Radiol. 2014;49:23-28.

20. Vande Velde G, De Langhe E, Poelmans J, et al. Magnetic resonance imaging for noninvasive assessment of lung fibrosis onset and progression: cross-validation and comparison of different magnetic resonance imaging protocols with micro-computed tomography and histology in the bleomycin-induced mouse model. Invest Radiol. 2014;49:691-698.

21. Eichinger M, Heussel CP, Kauczor HU, et al. Computed tomography and magnetic resonance imaging in cystic fibrosis lung disease. J Magn Reson Imaging. 2010;32:1370-1378.

22. Eichinger M, Optazaite DE, Kopp-Schneider A, et al. Morphologic and functional scoring of cystic fibrosis lung disease using MRI. Eur J Radiol. 2012;81: 1321-1329.

23. Hueper K, Parikh MA, Prince MR, et al. Quantitative and semiquantitative measures of regional pulmonary microvascular perfusion by magnetic resonance imaging and their relationships to global lung perfusion and lung diffusing 
capacity: the multiethnic study of atherosclerosis chronic obstructive pulmonary disease study. Invest Radiol. 2013;48:223-230.

24. Ingrisch M, Maxien D, Schwab F, et al. Assessment of pulmonary perfusion with breath-hold and free-breathing dynamic contrast-enhanced magnetic resonance imaging: quantification and reproducibility. Invest Radiol. 2014;49:382-389.

25. Frenzel T, Lengsfeld P, Schirmer H, et al. Stability of gadolinium-based magnetic resonance imaging contrast agents in human serum at $37^{\circ} \mathrm{C}$. Invest Radiol. 2008; 43:817-828.

26. Pietsch H, Raschke M, Ellinger-Ziegelbauer H, et al. The role of residual gadolinium in the induction of nephrogenic systemic fibrosis-like skin lesions in rats. Invest Radiol. 2011;46:48-56.

27. Renz DM, Durmus T, Böttcher J, et al. Comparison of gadoteric acid and gadobutrol for detection as well as morphologic and dynamic characterization of lesions on breast dynamic contrast-enhanced magnetic resonance imaging. Invest Radiol. 2014;49:474-484.

28. Hansell DM, Bankier AA, MacMahon H, et al. Fleischner Society: glossary of terms for thoracic imaging. Radiology. 2008;246:697-722.

29. Puderbach M, Eichinger M, Gahr J, et al. Proton MRI appearance of cystic fibrosis: comparison to CT. Eur Radiol. 2007;17:716-724.

30. Kaul D, Grupp U, Kahn J, et al. Reducing radiation dose in the diagnosis of pulmonary embolism using adaptive statistical iterative reconstruction and lower tube potential in computed tomography. Eur Radiol. 2014;24:2685-2691.

31. Bilton D, Canny G, Conway S, et al. Pulmonary exacerbation: towards a definition for use in clinical trials. Report from the EuroCareCF Working Group on outcome parameters in clinical trials. J Cyst Fibros. 2011;10:S79-S81.

32. Fuchs HJ, Borowitz DS, Christiansen DH, et al. Effect of aerosolized recombinant human DNase on exacerbations of respiratory symptoms and on pulmonary function in patients with cystic fibrosis. The Pulmozyme Study Group. $N$ Engl J Med. 1994;331:637-642.

33. Montella S, Santamaria F, Salvatore M, et al. Lung disease assessment in primary ciliary dyskinesia: a comparison between chest high-field magnetic resonance imaging and high-resolution computed tomography findings. Ital $J$ Pediatr. 2009;35:24.

34. Theilmann RJ, Arai TJ, Samiee A, et al. Quantitative MRI measurement of lung density must account for the change in $\mathrm{T} 2 *$ image with lung inflation. J Magn Reson Imaging. 2009;30:527-534.

35. Wheeler WB, Williams M, Matthews WJ Jr, et al. Progression of cystic fibrosis lung disease as a function of serum immunoglobulin G levels: a 5-year longitudinal study. J Pediatr. 1984;104:695-699.

36. Garside JP, Kerrin DP, Brownlee KG, et al. Immunoglobulin and IgG subclass levels in a regional pediatric cystic fibrosis clinic. Pediatr Pulmonol. 2005;39: 135-140.

37. Kerem E, Reisman J, Corey M, et al. Prediction of mortality in patients with cystic fibrosis. N Engl J Med. 1992;326:1187-1191.

38. de Jong PA, Nakano Y, Lequin MH, et al. Progressive damage on high resolution computed tomography despite stable lung function in cystic fibrosis. Eur Respir J. 2004;23:93-97.

39. Gordic S, Morsbach F, Schmidt B, et al. Ultralow-dose chest computed tomography for pulmonary nodule detection: first performance evaluation of single energy scanning with spectral shaping. Invest Radiol. 2014;49:465-473.

40. Chae EJ, Kim N, Seo JB, et al. Prediction of postoperative lung function in patients undergoing lung resection: dual-energy perfusion computed tomography versus perfusion scintigraphy. Invest Radiol. 2013;48:622-627. 